07,11

\title{
Влияние дислокаций на структурный параметр порядка в кристалле при упругой деформации кручения
}

\author{
(С Ю.Д. Заворотнев ${ }^{1}$, А.Ю. Захаров ${ }^{2,}$, Л.С. Метлов ${ }^{1,3}$ \\ ${ }^{1}$ Донецкий фризико-технический институт им. А.А. Галкина, \\ Донецк, Украина \\ ${ }^{2}$ Новгородский государственный университет им. Ярослава Мудрого, \\ Великий Новгород, Россия \\ ${ }^{3}$ Донецкий национальный университет, \\ Донецк, Украина \\ ๑E-mail: A.Yu.Zakharov@gmail.com \\ (Поступила в Редакцию 11 апреля 2017 г.)
}

\begin{abstract}
Изучено взаимодействие структурной подсистемы с дислокациями при наложении упругой деформации кручения. Рассмотрение проведено в рамках феноменологической теории Ландау при отказе от приближения постоянства модулей неприводимых векторов. Показано появление дополнительных пространственных амплитудных и частотных осцилляций, связанных с изменением модуля структурного параметра порядка и плотности дислокаций.
\end{abstract}

А.Ю. Захаров признателен Министерству образования и науки РФ за финансовую поддержку в рамках госзадания (грант 3.3572.2017/ПЧ).

DOI: 10.21883/FTT.2017.11.45073.121

\section{1. Введение}

В твердых телах имеет место множество фазовых переходов (ФП) различной природы. Наиболее распространенными являются структурные ФП, при которых меняется, в частности, симметрия кристалла. В рамках феноменологической теории Ландау такие ФП описываются с помощью параметра порядка (ПП). Последние в случае структурного ФП представляют собой линейные комбинации смещений ионов в элементарной ячейке и преобразуются по некоторому неприводимому представлению группы симметрии кристалла. Структурные ФП в силу существования различных взаимодействий всегда сопровождаются изменениями в других кристаллических подсистемах, в частности магнитной [1]. При рассмотрении таких ситуаций часто затруднительно определить, изменения какой из подсистем являются первичными, а какой - вторичными. Этот вопрос легко решается, если изменения одной из подсистем вызваны внешним воздействием. Подобная задача рассматривалась в работах [2-5], где изучалось взаимодействие структурной и магнитной подсистем при наложении интенсивной пластической деформации кручением при отказе от приближения постоянства модулей всех ПП. Предполагалось, что изученные процессы осуществляются в идеальных кристаллах. Однако структурные ФП могут быть осложнены наличием дефектов кристаллической решетки, например, дислокаций. Последние могут быть охарактеризованы плотностью дислокаций, которая будет играть роль соответствующего ПП [6]. В силу определения такой ПП преобразуется по полносимметричному представлению группы симметрии кристалла. Кинетика дислокационной подсистемы может быть описана в рамках неравновесной эволюционной термодинамики [6,7]. Взаимодействие структурного ПП с дислокационной подсистемой описывается перекрестными слагаемыми в термодинамическом потенциале.

Представляет интерес влияние на распределение модуля структурного ПП наличия дислокаций, а также обратное влияние кристаллографического ПП на распределение и организацию дислокаций при наложении упругой деформации кручения (УДК). Ниже будет показано, что в этой задаче за счет такого влияния возможно образование периодического расположения дислокаций в пространстве так называемой сверхрешетки.

\section{2. Теория}

Пусть в модельном кристалле с дислокациями при понижении температуры имеет место структурный фазовый переход, который может быть как первого, так и второго рода. Рассмотрим поведение соответствующего ПП вблизи и вдали от этих ФП при наложении винтового крутящего упругого момента вдоль оси OZ. При таком воздействии не происходит какой-либо ФП, но возникает пространственная периодическая спиральная структура. В силу наличия взаимодействия между структурным ПП и дефектами наложение УДК должно оказать воздействие и на плотность дислокаций. Поставленная задача сводится к изучению взаимодействия между дефектным и структурным ПП. Дефектным ПП является плотность дислокаций. Структурный ПП линейная комбинация отклонений ионов элементарной 

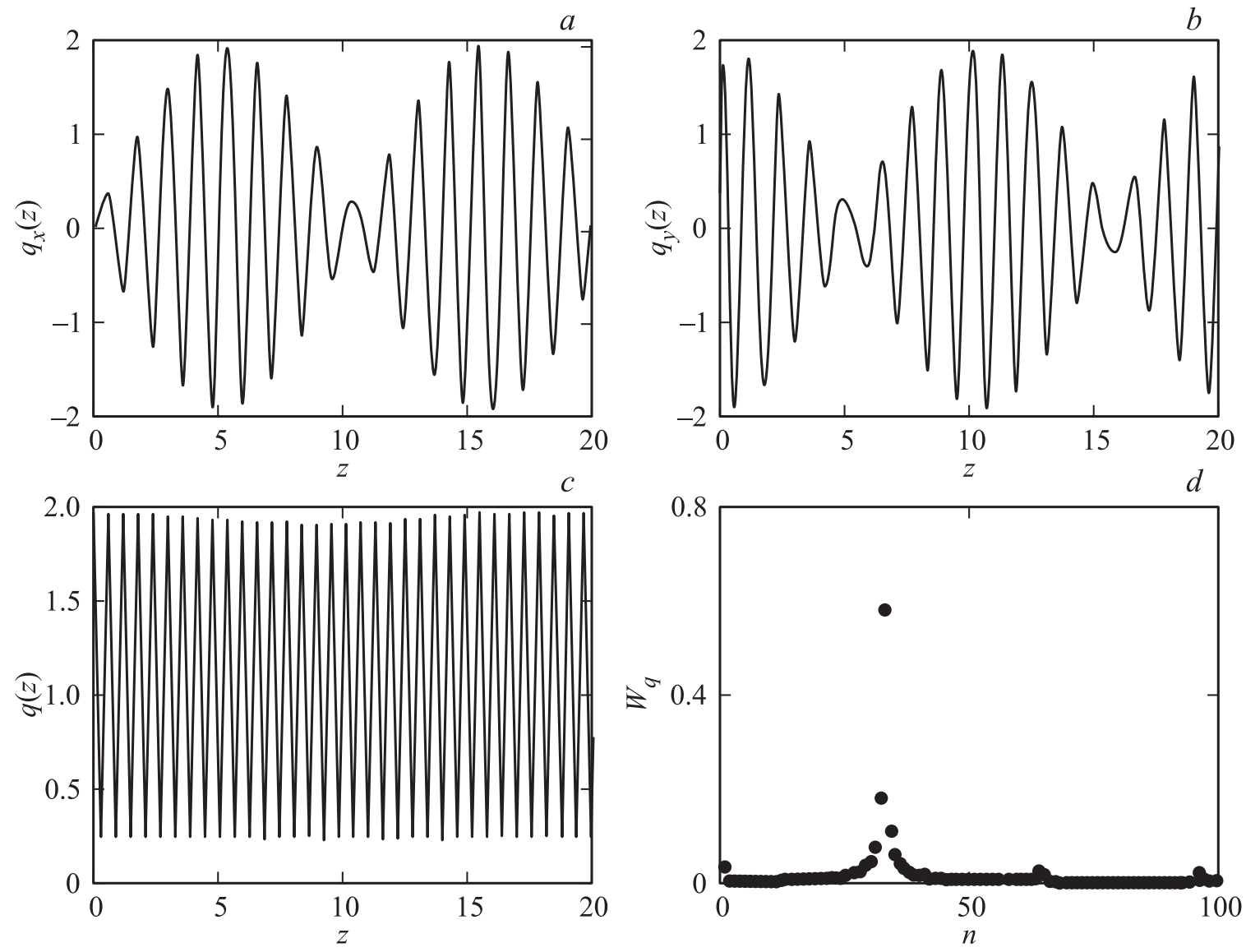

Рис. 1. Пространственные зависимости структурного ПП при ФП второго рода в идеальном кристалле. $d-$ амплитуда первой гармоники уменьшена в 50 раз.

ячейки - векторная величина. Компоненты последнего ПП преобразуются по соответствующим неприводимым представлениям группы симметрии кристалла. Дефектный ПП по определению является скаляром (инвариантом относительно любой операции симметрии). Поэтому в разложении неравновесного термодинамического потенциала (НТДП) в ряд по этому ПП необходимо оставлять не только четные, но и нечетные слагаемые. Предположим, что симметрия кристалла разрешает существование инвариантов Лифшица. Тогда в рамках феноменологической теории Ландау плотность НТДП запишется в виде:

$$
\begin{aligned}
\Phi= & \frac{\beta_{1}}{2} q^{2}+\frac{\beta_{2}}{4} q^{4}+\frac{\beta_{3}}{6} q^{6}-\gamma_{1} M^{r}\left(q_{x} \frac{\partial q_{y}}{\partial z}-q_{y} \frac{\partial q_{x}}{\partial z}\right) \\
& +\gamma_{2} M^{s}\left[\left(\frac{\partial q_{x}}{\partial z}\right)^{2}+\left(\frac{\partial q_{y}}{\partial z}\right)\right]+\gamma_{3} q^{2} h+\gamma_{4} q^{2} h^{2} \\
& +\gamma_{5}\left(\frac{\partial h}{\partial z}\right)^{2}+\varphi_{1} h+\frac{\varphi_{2}}{2} h^{2}+\frac{\varphi_{3}}{3} h^{3}+\frac{\varphi_{4}}{4} h^{4}
\end{aligned}
$$

где $\beta_{i}(i=1-3), \gamma_{i}(i=1-5), \varphi_{i}(i=1-4)-$ феноменологические коэффициенты, $h, q$ - дефектный и струк- турный ПП соответственно, $M$ - модуль крутящего момента, $r, s-$ показатели степеней, по данным работы [1], $r=6, s=2$. Слагаемые с коэффициентами $\gamma_{3}$, $\gamma_{4}$ описывают различные виды взаимодействий двух ПП. Случай $\gamma_{5}=0$ описывает равномерное распределение дефектов в кристалле, в противном случае имеет место пространственное изменение соответствующего ПП. В нашей модели описание магнитной спиральной структуры [8] распространено на кристаллическую структуру, возникающую при УДК. Слагаемые с пространственными производными описывают винтовую деформацию (несоразмерная длиннопериодическая спиральная структура) и содержат пропорциональный моменту множитель, т. е. при $M=0$ отсутствует пространственная спиральная структура. Константы $\varphi_{i}(i=1-4)$ не зависят от температуры, так как отсутствует ФП по температуре с образованием дислокаций. В потенциале (1) следует учесть также упругое взаимодействие. Переменные, описывающие это взаимодействие, можно исключить, определяя с помощью уравнений состояний их равновесные значения в зависимости от величины структурного ПП. После подстановки найденных выражений в потенциал получаем новый НТДП. Он формально будет совпадать с НТДП без учета упругого взаимодействия. При 



Рис. 2. Пространственные зависимости компонент структурного ПП при наличии взаимодействия (ФП второго рода).


Рис. 3. Пространственная зависимость структурного ПП при наличии дислокаций и их слабом взаимодействии (ФП первого рода). $b$ - амплитуда первой гармоники уменьшена в 10 раз.

этом новые константы будут зависеть от температуры и давления. В дальнейшем будем предполагать, что процедура исключения проведена. При отказе от предположения постоянства модулей неприводимых векторов в случае однородного распределения дефектов получаем следующую дифференциально-алгебраическую систему уравнений Эйлера:

$$
\left\{\begin{array}{l}
2 \gamma_{2} M^{s} \frac{\partial^{2} q_{x}}{\partial z^{2}}+\gamma_{1} M^{r} \frac{\partial q_{y}}{\partial z} \\
\quad-q_{x}\left(\beta_{1}+\beta_{2} q^{2}+\beta_{3} q^{4}+2 \gamma_{3} h+2 \gamma_{4} h^{2}\right)=0 \\
2 \gamma_{2} M^{s} \frac{\partial^{2} q_{y}}{\partial z^{2}}-\gamma_{1} M^{r} \frac{\partial q_{x}}{\partial z} \\
\quad-q_{y}\left(\beta_{1}+\beta_{2} q^{2}+\beta_{3} q^{4}+2 \gamma_{3} h+2 \gamma_{4} h^{2}\right)=0 \\
\varphi_{1}+\varphi_{2} h+\varphi_{3} h^{2}+\varphi_{4} h^{3}+\gamma_{3} q^{2}+2 \gamma_{4} q^{2} h=0
\end{array}\right.
$$

Если распределение дефектов в кристалле неоднородно, то соответствующая система уравнений имеет вид:

$$
\left\{\begin{aligned}
& 2 \gamma_{2} M^{s} \frac{\partial^{2} q_{x}}{\partial z^{2}}+\gamma_{1} M^{r} \frac{\partial q_{y}}{\partial z} \\
&-q_{x}\left(\beta_{1}+\beta_{2} q^{2}+\beta_{3} q^{4}+2 \gamma_{3} h+2 \gamma_{4} h^{2}\right)=0 \\
& 2 \gamma_{2} M^{s} \frac{\partial^{2} q_{y}}{\partial z^{2}}- \gamma_{1} M^{r} \frac{\partial q_{x}}{\partial z} \\
&-q_{y}\left(\beta_{1}+\beta_{2} q^{2}+\beta_{3} q^{4}+2 \gamma_{3} h+2 \gamma_{4} h^{2}\right)=0 \\
& 2 \gamma_{s} \frac{\partial^{2} h}{\partial z^{2}}-\left(\varphi_{1}+\varphi_{2} h+\varphi_{3} h^{2}\right. \\
&\left.+\varphi_{4} h^{3}+\gamma_{3} q^{2}+2 \gamma_{4} q^{2} h\right)=0
\end{aligned}\right.
$$

Везде в дальнейшем будем предполагать, что $\varphi_{1}>0$, $\varphi_{2}<0, \varphi_{3}>0, \varphi_{4}<0$. Системы (2) и (3) решить аналитически затруднительно. Поэтому с помощью математического пакета MathCad 15 был проведен численный 


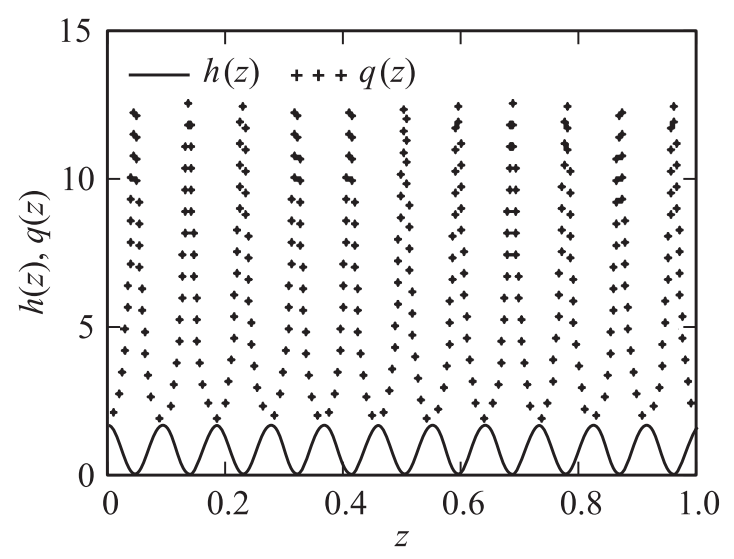

Pис. 4. Поведение структурного и дислокационного ПП при ФП первого рода и больших значениях модуля их взаимодействия.

анализ. При этом также был проведен Фурье-анализ полученных решений, который позволил выявить наличие кратных и некратных гармоник.

\section{3. Обсуждение результатов}

Поскольку предполагается, что структурная подсистема находится в упорядоченном состоянии, то в настоящем разделе, согласно теории Ландау, предполагается $\beta_{1}<0, \beta_{3}>0$. Коэффициент $\beta_{2}$ определяет род ФП, поэтому в каждом пункте этого раздела его знак будет оговариваться отдельно.

Рассмотрим случай однородного распределения дислокаций [система (2)]. Пусть $\gamma_{3}=0, \gamma_{4}=0$.

1) $\beta_{2}>0$. В этом случае имеет место структурный ФП второго рода. Как следует из третьего (алгебраического) уравнения системы (2), характер изменения величины плотности дислокаций зависит от изменения амплитуды модуля структурного ПП. Поэтому качественное изменение обеих величин будет одинаковым и иллюстрации в дальнейшем будут приведены только для зависимости $q(z)$. При отсутствии дислокаций при данных значениях коэффициентов термодинамического потенциала график зависимости $q(z)$ будет практически синусоидальным (рис. 1,c), причем имеет место слабая двухсторонняя синфазная пространственная амплитудная модуляция. Анализ Фурье (рис. 1,d) показывает, что существенными являются гармоники, расположенные в окрестностях 35, 70 и 100-й. Поведение составляющих $q_{x}(z)$ и $q_{y}(z)$ существенно отличаются от поведения модуля ПП (рис. $1, a, b)$ и представляет собой колебания с большой симметричной противофазной амплитудной модуляцией. При малых значениях крутящего момента появляется колебательный процесс $h(z)$, и уменьшается число колебаний $q_{x}(z)$ и $q_{y}(z)$ на протяжении периода амплитудной модуляции. С ростом $\left|\gamma_{4}\right|$ неравномерность колебаний модуля структурного ПП и $h(z)$ становятся меньше, что приводит к уменьшению амплитуд гармоник соответствующих разложений Фурье. Дальнейшее увеличение $\left|\gamma_{4}\right|$ приводит к появлению сдвинутых по фазе амплитудных модуляций величин $q_{x}(z)$ и $q_{x}(z)$ (рис. $\left.2, a, b\right)$. Однако эти изменения слабо влияют на поведение модуля структурного ПП, но растет постоянная составляющая $h$ и размах ее колебаний. Анализ Фурье показывает, что гармоникой с самой большой амплитудой является 24-я.

2) $\beta_{2}<0$. По температуре происходит структурный ФП первого рода. При отсутствии взаимодействия между дислокациями и структурным параметром порядка колебательный процесс модуля структурного ПП имеет периодический характер с острой вершиной (рис. 3,a). График амплитуд анализа Фурье представлен на рис. $3, b$. Максимумы плотности дислокаций приходятся на минимумы колебаний структурного ПП. При увеличении величины $\left|\gamma_{4}\right|$ график изменений дислокационного ПП опускается вниз, и при некоторой величине $\left|\gamma_{4}\right|$ значения минимумов колебаний дефектного ПП становятся равными нулю (рис. 4). Это означает, что дислокации будут группироваться в окрестностях минимумов колебаний структурного ПП. Такие группы будут образовывать периодическую одномерную решетку дислокаций. С ростом величины момента $M$ период такой решетки растет,


Рис. 5. Поведение структурного и дислокационного ПП при ФП первого рода $\left(\gamma_{4}=\gamma_{5}=0\right)$. $\gamma_{3}<0(a), \gamma_{3}>0(b)$. 


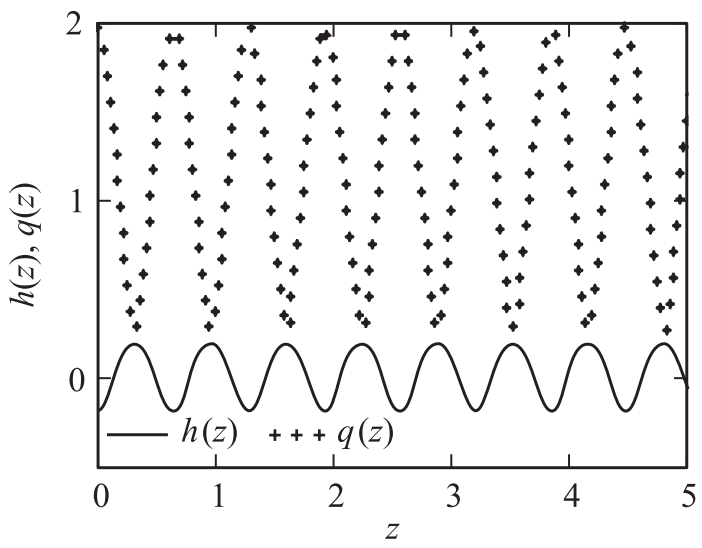

Рис. 6. Поведение структурного и дислокационного ПП при ФП второго рода $\left(\gamma_{4}=\gamma_{5}=0, \gamma_{3}<0\right)$.

и области концентрации и отсутствия дислокаций увеличиваются в размерах.

Рассмотрим случай однородного распределения дислокаций с другим типом взаимодействия: $\gamma_{4}=0, \gamma_{3}<0$.

1) $\beta_{2}<0$. Такой знак коэффициента НТДП описывает ФП первого рода структурной подсистемы. Измене- ния структурного и дислокационного ПП показаны на рис. 5, $a$. Видно, что на зависимости имеются участки с отрицательными значениями $h(z)$. Эти решения являются нефизическими. Следовательно, в таких областях при невысокой концентрации плотность дислокаций должна быть равна нулю. Это означает появление бездислокационных зон и создание дислокационной решетки. Изменение концентрации происходит очень резко, что приводит к появлению структуры типа доменной с тонкими стенками и однородной плотностью в большем промежутке. При уменьшении модуля взаимодействия размеры бездислокационных зон уменьшаются. Увеличение количества дислокаций приводит к росту постоянной составляющей [сдвиг кривой $h(z)$ вверх] и уменьшению пространственной длины бездислокационных областей. При положительном знаке взаимодействия $\left(\gamma_{3}>0\right)$ решение для $h(z)$ во всех пространственных точках всегда физично (рис. 5, $b$ ). Следовательно, в этом случае возникает только пространственно осциллирующее распределение дислокационного ПП.

2) $\beta_{2}>0$. Имеем ФП второго рода в структурной подсистеме. Пространственные зависимости двух ПП при наложении кручения показаны на рис. 6,a. Видно, что зависимость $h(z)$ практически пространственно
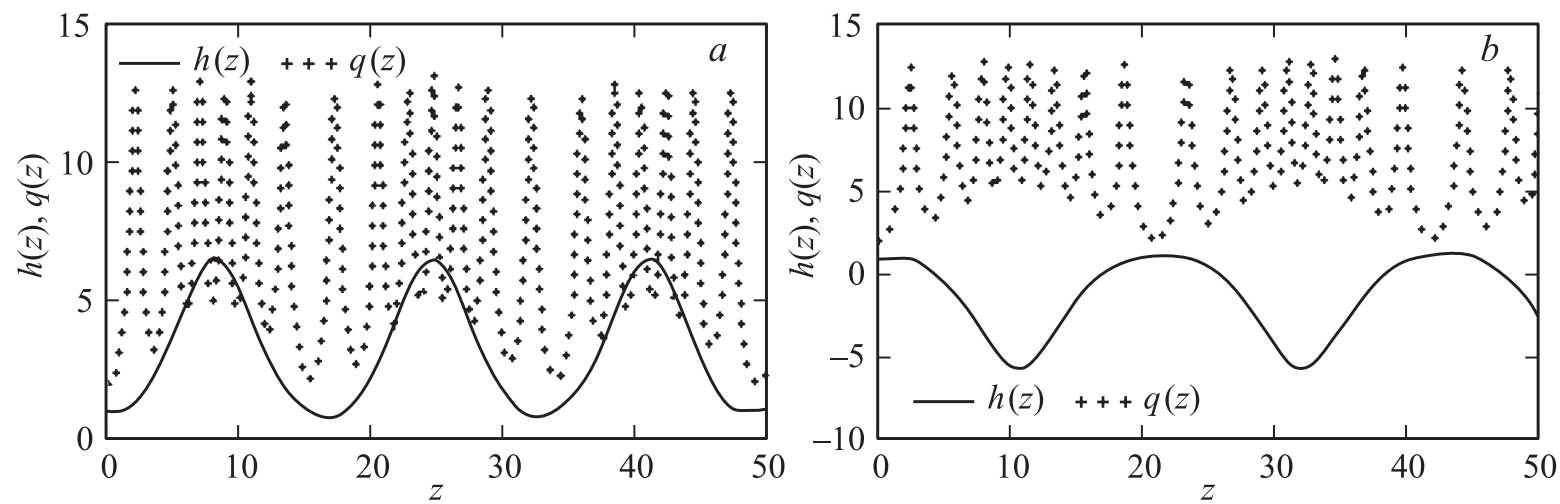

Рис. 7. Поведение структурного и дислокационного ПП при ФП первого рода и синусоидальном распределении дефектов. $\gamma_{3}<0$, $\gamma_{5} \neq 0, \gamma_{4}=0(a), \gamma_{3}>0, \gamma_{5} \neq 0, \gamma_{4}=0(b)$.
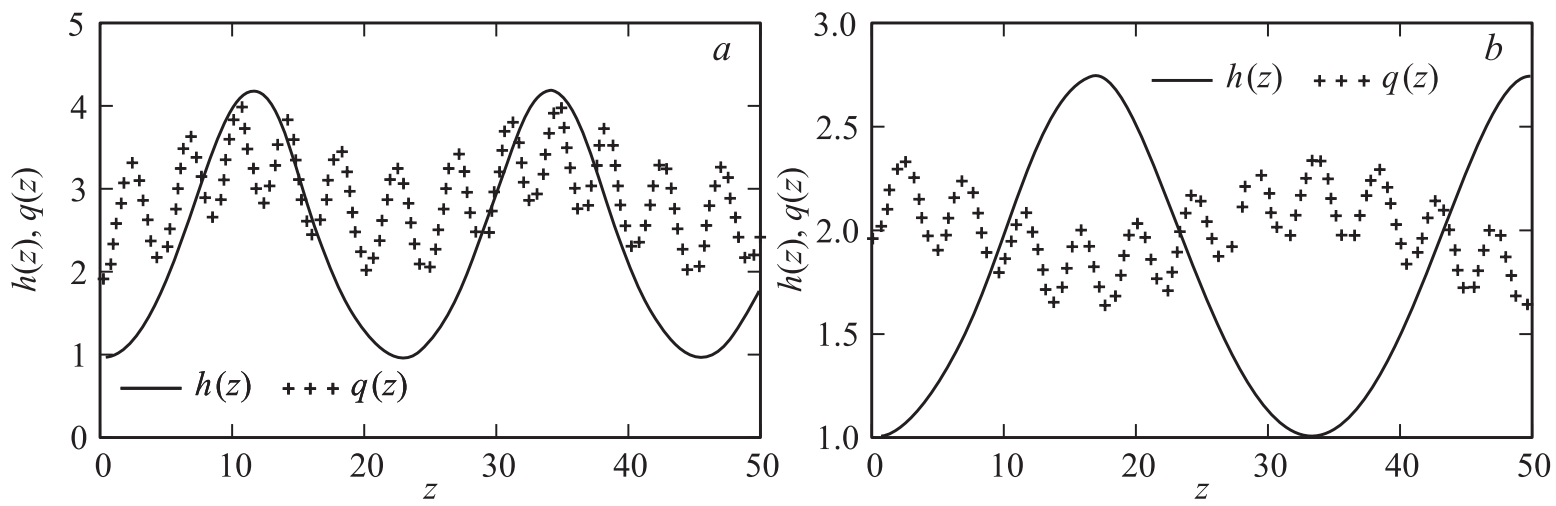

Рис. 8. Поведение структурного и дислокационного ПП при ФП второго рода и синусоидальном распределении дислокаций. $\gamma_{3}<0, \gamma_{5} \neq 0, \gamma_{4}=0(a), \gamma_{3}>0, \gamma_{5} \neq 0, \gamma_{4}=0(b)$. 
синусоидальна. Кривые обоих ПП являются пространственно противофазными. Так же как и в случае 1), эта зависимость имеет нефизические решения, в интервалах которых плотность дислокаций равна нулю. Однако длины дислокационных и бездислокационных участков практически одинаковы. Следовательно, примерно половина кристалла очищается от дислокаций, и решетка на этих участках становится идеальной. Изменение знака взаимодействия, так же как и в предыдущем случае, приводит к сдвигу кривой $h(z)$ вверх и исчезновению нефизических решений. Зависимости $h(z)$ и $q(z)$ становятся синфазными.

Пусть в кристалле создано неоднородное распределение дислокаций, которое подчиняется синусоидальному закону, период которого сравним с периодом внешнего кручения [система $(3), \gamma_{5} \neq 0$ ].

1) $\beta_{2}<0, \quad \gamma_{3}<0$. В структурной подсистеме ФП первого рода. Пространственные зависимости $h(z)$ и $q(z)$ (рис. 7,a) синфазны. Взаимодействие обусловливает взаимное усиление колебательных процессов. При этом появляются постоянные составляющие, что обусловливает увеличение средней плотности дислокаций, но отсутствуют области нефизических решений. При изменении знака взаимодействия (рис. 7,b), колебательные процессы становятся противофазными и происходит взаимное „отталкивание“ между ПП. При этом появляются области, свободные от дислокаций.

2) $\beta_{2}>0, \gamma_{3}<0$. Имеем ФП второго рода. Соответствующие пространственные зависимости $h(z)$ и $q(z)$ приведены на рис. $8, a$. По сравнению с предыдущими вариантами здесь сильно возрастает амплитуда пространственных колебаний плотности дислокаций. Кроме колебаний $q(z)$, возникших благодаря отказу от приближения постоянства модулей ПП, появляется колебание, синфазное с изменением дислокационного ПП. Зоны бездислокационности отсутствуют. При изменении знака взаимодействия указанные колебания становятся противофазными (рис. $8, b$ ). Зоны бездислокационности также отсутствуют.

\section{4. Заключение}

Показана возможность изучения взаимодействия векторного (структурный ПП) и скалярного (плотность дислокаций) полей с использованием формализма Ландау при отказе от приближения постоянства модулей неприводимых векторов. Результаты взаимодействия двух ПП существенно различаются при структурных ФП первого и второго родов. Путем варьирования температуры и величины крутящего момента можно формировать различные распределения изменений модулей ПП. При малых концентрациях дислокаций возможно появление бездислокационных зон.

\section{Список литературы}

[1] Ю.Д. Заворотнев. ФНТ 39, 178 (2013).

[2] Yu.D. Zavorotnev, E.H. Pashinska, V.N. Varyukhin, O.Yu. Popova. J. Magn. Magn. Mater. 349, 244 (2014).

[3] Ю.Д. Заворотнев, Е.Г. Пашинская, В.Н. Варюхин. Изв. РАН. Сер. физ. 78, 1011 (2014).

[4] Ю.Д. Заворотнев, Е.Г. Пашинская. ФНТ 40, 1237 (2014).

[5] Ю.Д. Заворотнев, Е.Г. Пашинская. ФТТ 58, 647 (2016).

[6] L.S. Metlov. Phys. Rev. Lett. 106, 165506 (2011).

[7] L.S. Metlov. Phys. Rev. E 90, 022124 (2014).

[8] И.Е. Дзялошинский. ЖЭТФ 46, 1420 (1964). 